\title{
Protección social y nivel de salud de los trabajadores de la economía informal
}

\author{
MARISOL CONCHA(1), JAVIER LABBÉ(2), MARLENE SÁNCHEZ(3)
}

El concepto de sector informal' ha sido objeto de innumerables controversias debido a la heterogeneidad del fenómeno. En el año 2002, como resultado de una serie de investigaciones la OIT comienza a utilizar la expresión de "economía informal" en lugar de "sector informal" para denominar a este fenómeno. La definición de "economía informal" se centra en las actividades informales y en las relaciones de trabajo.

Múltiples hipótesis explican la existencia de la economía informal, pudiéndose distinguir tres líneas de pensamiento. Algunas hipótesis, consideran que es el resultado de la influencia de variables sociales y demográficas; otras, que es el resultado de variables económicas (crecimiento, salario mínimo y otros) y, otras, que es el resultado de los costos que imponen las regulaciones que afectan al trabajo formal. Cualquiera sea la causa, en Chile la pertenencia a este sector de la economía se traduce en ahorros en el costo laboral que, como mínimo, equivalen a 25 puntos porcentuales del salario: 13 se obtendrían de la evasión del pago de las cotizaciones para la previsión de vejez; 7 mediante la evasión del pago de las cotizaciones para la previsión de salud; 3 puntos porcentuales se obtendrían por razón de la evasión del pago de las cotizaciones para el seguros de desempleo; y 2 , a través de la evasión de las cotizaciones para el seguro de accidentes laborales ${ }^{2}$. No obstante lo anterior, la evasión de las regulaciones también conlleva costos. En efecto, en muchos casos las actividades informales están sujetas a penas, multas y confiscaciones, cuando son pesquisadas por la autoridad y falta de acceso al mercado de capitales. Adicionalmente, estos trabajadores carecen de previsión social para la vejez y de seguro de desempleo, además la protección a la salud está limitada a los indigentes y usualmente se desempeñan en condiciones laborales no reguladas.

Según la Encuesta Casen 2000, alrededor de dos millones y medio de personas en Chile se desempeñaría en la economía informal, lo que representa aproximadamente un $46,6 \%$ del total de trabajadores ocupados en el año 20003. Alrededor de 1.404 .992 trabajadores tendría acceso a cobertura médica, en el caso de enfermedad común, maternidad o accidente del trabajo, sin derecho al subsidio por incapacidad laboral. El resto de estos trabajadores estaría afiliado a Fonasa en los grupos B, C y D (31,4 puntos porcentuales) y a las isapres, como independientes (con derecho a SIL) o pensionados (7,6 puntos porcentuales $)^{4}$. De manera, adicional, aproximadamente un $79,4 \%$ de los trabajadores no tendría sistema de previsión para la vejez. El resto, cotizaría como independiente en alguna AFP (17,2 puntos porcentuales) o sería pensionado del INP $(2,3$ puntos porcentuales) ${ }^{5}$. (Tabla I).

Un análisis de los efectos de la pertenencia a la economía informal sobre el nivel de salud, las atenciones de salud recibidas por los trabajadores y la calidad de las mismas, basada en la Encuesta Casen 2000 muestra que:

\footnotetext{
(1) Gerencia Salud. Asociación Chilena de Seguridad.

(2) Instituto de Políticas Públicas y Gestión en Salud, Salud y Futuro. UNAB.

(3) Asesor Independiente.
} 
Tabla 1. Número y distribución porcentual de los trabajadores de la economía informal según acceso a la protección social. Chile; 2000

\begin{tabular}{|c|c|c|}
\hline Asistencia médica ${ }^{(6)}$ & 1.404 .992 & 58,4 \\
\hline Prestaciones de enfermedad(6) & 1.404 .992 & 58,4 \\
\hline Maternidad $(6)$ & 1.404 .992 & 58,4 \\
\hline Accidentes laborales( $(7)$ & 1.404 .992 & 58,4 \\
\hline Vejez & 1.910 .543 & 79,4 \\
\hline Invalidez & 1.910 .543 & 79,4 \\
\hline Sobrevivientes & 1.910 .543 & 79,4 \\
\hline Desempleo & 2.407 .351 & 100,0 \\
\hline Circunstancias familiares & 2.407 .351 & 100,0 \\
\hline
\end{tabular}

Fuente: Casen, 2000

- Los trabajadores informales presentan una situación desmejorada en relación con los trabajadores formales del país. En efecto, un $13,5 \%$ de los trabajadores formales calificó su salud como "Muy buena", en cambio sólo un $10,5 \%$ de los informales la consideró así. Por otra parte, un 1,9\% de los trabajadores formales consideró su salud como "Mala" o "Muy mala", en cambio un $4,7 \%$ de los informales la calificó en las mismas categorías (Figura 1). En promedio, Ios trabajadores formales calificaron su salud con nota 5,3, en una escala de 1 a 7 , mientras que los trabajadores informales la clasificaron con nota 5,1. Estas diferencias resultaron estadísticamente significativas.

- Los trabajadores informales presentan un

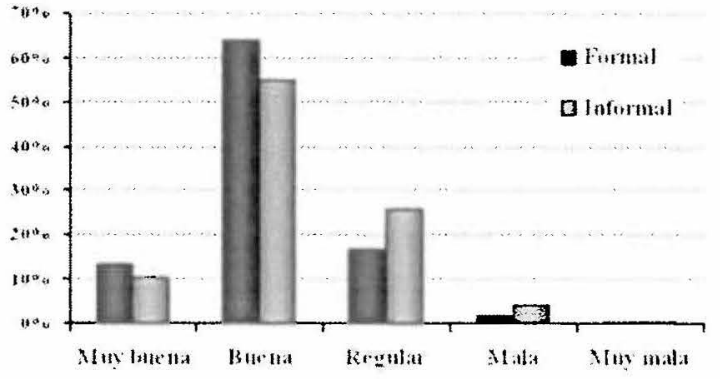

Figura 1: Usted diría que su salud general es...

menor nivel de acceso a hospitalizaciones e intervenciones quirúrgicas. Es así como, aproximadamente un $4,3 \%$ de los trabajadores formales fue hospitalizado por una causa no relacionada con parto, embarazo o cesárea ${ }^{9}$, en cambio, sólo un $3,6 \%$ de los trabajadores informales fue hospitalizado por estas mismas causas. Este resultado, podría tener una interpretación ambigua, ya que, la menor tasa de hospitalización de los trabajadores informales podría estar relacionada con un mejor nivel de salud, no obstante, los resultados anteriores permiten descartar esta hipótesis. Por último, los trabajadores informales sienten que recibieron atenciones de menor calidad, en términos de oportunidad y de la satisfacción en cuanto

Tabla 2. ¿Usted considera que la hospitalización, en relación al momento en que ésta le fue indicada?

\begin{tabular}{|c|c|c|c|c|c|c|c|c|c|}
\hline \multirow[b]{3}{*}{ I Oportuna } & \multicolumn{5}{|c|}{ Trabajador formal } & \multicolumn{4}{|c|}{ Trabajador informal } \\
\hline & & porción & & rror $(1)$ & Desviación(2) & Proporción & & Error (1) & Desviación(2) \\
\hline & $($ nota 7$)$ & $91,0 \%$ & \pm & $1,3 \%$ & $0,7 \%$ & $85,9 \%$ & \pm & $1,6 \%$ & $0,9 \%$ \\
\hline 2 Con alguna demora & $($ nota 4$)$ & $5,1 \%$ & \pm & $1,0 \%$ & $2,0 \%$ & $7,7 \%$ & \pm & $1,2 \%$ & $2,0 \%$ \\
\hline 3 Tardía & $($ nota 1$)$ & $2,9 \%$ & \pm & $0,7 \%$ & $2,0 \%$ & $5,0 \%$ & \pm & $1,0 \%$ & $2,6 \%$ \\
\hline 4 No sabe & & $1,0 \%$ & \pm & $0,4 \%$ & $2,6 \%$ & $1,4 \%$ & \pm & $0,5 \%$ & $2.4 \%$ \\
\hline Nota promedio & & 6,7 & \pm & 0,058 & 1,313 & 6.5 & \pm & 0,063 & 1,388 \\
\hline
\end{tabular}

(1) El margen de error se estimó con un nivel de significancia de $95 \%$.

(2) Desviación estándar poblacional.

1 También se le ha denominado sector oculto, paralelo, no moderno, clandestino, invisible, negro.

2 Según cálculos efectuados por la Asociación Chilena de Seguridad, la tasa de cotización promedio para el Seguro de Accidentes del Trabajo, asciende a $1,75 \%$.

3 Sánchez M. y Labbé J. "El sector informal en Chile: una visión estadística”. Santiago de Chile. 2004.

4 A partir de la Encuesta Casen, no se ha podido establecer la situación del 2,6\% restante de los trabajadores.

5 A partir de la Encuesta Casen, no se ha podido establecer la situación del $1,1 \%$ restante de los trabajadores. 
Tabla 3. ¿Qué grado de satisfacción obtuvo por la atención recibida durante su última hospitalización, en atención médica?

\begin{tabular}{|c|c|c|c|c|c|c|c|c|}
\hline & \multicolumn{5}{|c|}{ Trabajador formal } & \multicolumn{3}{|c|}{ Trabajador informal } \\
\hline & & oporción & & trror ${ }^{(1)} \mathrm{I}$ & $\operatorname{Desviación}^{(2)}$ & Proporción & Error $^{(1)}$ & Desviación $\mathbf{n}^{(2)}$ \\
\hline 1 Muy bueno & $(\operatorname{nota} 7,0)$ & $45,2 \%$ & \pm & $2,9 \%$ & $2,3 \%$ & $38,3 \%$ & $\pm \quad 2,7 \%$ & $2,4 \%$ \\
\hline 2 Bueno & $($ nota 5,5$)$ & $48,2 \%$ & \pm & $2,9 \%$ & $2,0 \%$ & $53,9 \%$ & $\pm \quad 2,7 \%$ & $1,8 \%$ \\
\hline 3 Regular & $($ nota 4,0$)$ & $3,9 \%$ & \pm & $1,1 \%$ & $2,8 \%$ & $4,5 \%$ & $\pm \quad 1,1 \%$ & $2,7 \%$ \\
\hline 4 Malo & $(\operatorname{nota} 2,5)$ & $1,2 \%$ & \pm & $0,6 \%$ & $2,6 \%$ & $1,1 \%$ & $\pm \quad 0,6 \%$ & $2,3 \%$ \\
\hline 5 Muy malo & (nota 1,0$)$ & $0,6 \%$ & \pm & $0,4 \%$ & $2,9 \%$ & $1,1 \%$ & $\pm \quad 0,6 \%$ & $3,6 \%$ \\
\hline 6 No sabe & & $0,9 \%$ & \pm & $0,5 \%$ & $3,9 \%$ & $1,0 \%$ & $\pm 0,6 \%$ & $2,5 \%$ \\
\hline Nota promedio & & 6,1 & \pm & 0,059 & 1,025 & 5,9 & $\pm 0,055$ & 1,004 \\
\hline
\end{tabular}

(1) El margen de error se estimó con un nivel de significancia de $95 \%$.

(2) Desviación estándar poblacional.

Tabla 4. ¿Qué grado de satisfacción obtuvo por la atención recibida durante su última hospitalización, en trato de personal administrativo?

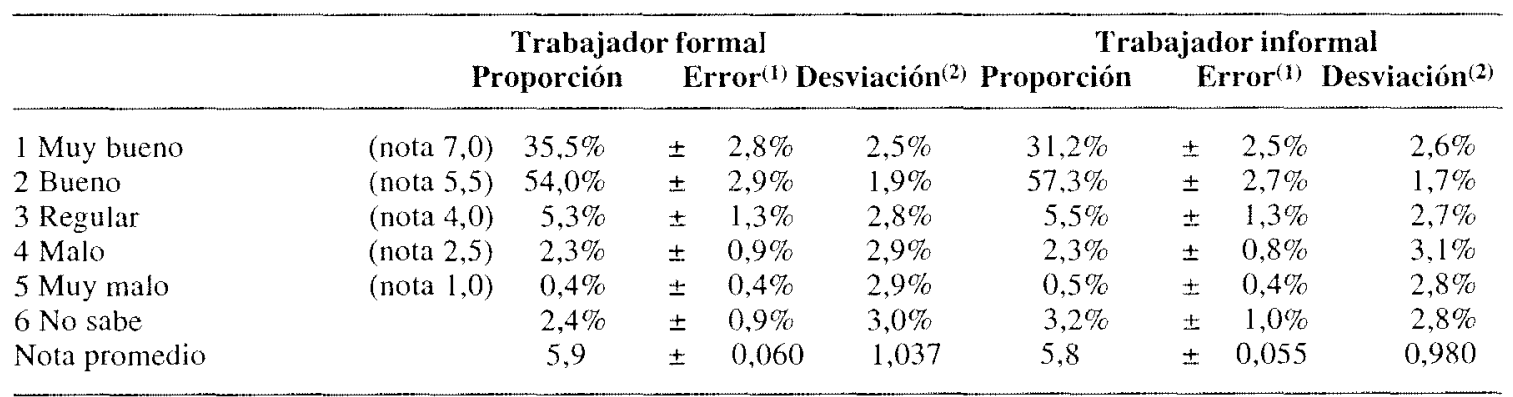

(1) El margen de error se estimó con un nivel de significancia de $95 \%$.

(2) Desviación estándar poblacional.

a la atención médica, el trato administrativo y la infraestructura.

En cuanto a la oportunidad, un $91 \%$ de los trabajadores formales afirmó que su hospitalización fue oportuna, en cambio sólo un $85,9 \%$ de los trabajadores informales afirmó lo mismo. Asimismo, un 2,9\% de los trabajadores formales aseveró que su hospitalización fue tardía y un $5 \%$ de los opinó lo mismo. (Tablas 2 y 5 ).
- En cuanto a la satisfacción con la atención médica, un $45,2 \%$ de los trabajadores formales manifestó que la atención médica fue "Muy buena", en cambio, sólo un 38,3\% de los trabajadores informales manifestó que la atención médica fue "Muy buena". En promedio los trabajadores formales calificaron la atención médica con nota 6,1 y los trabajadores informales la calificaron

6 Los trabajadores informales tendrían acceso y derecho a atenciones, prestaciones y maternidad como indigente en el Fonasa o como voluntario en Isapre, a pero no tendrían derecho ni acceso a subsidio por incapacidad laboral.

7 Los trabajadores informales tendrían acceso a prestaciones en el caso de accidentes laborales, ya sea como indigentes en Fonasa o como voluntario en isapre pero en ambos casos no tendría acceso a subsidio por incapacidad laboral. Cabe destacar que estas personas estarian haciendo uso de un sistema cuya prioridad y objetivo es atención prestacional de enfermedades comunes y derivadas de la maternidad.

8 Ministerio de Planificación y Cooperación, Gobiemo de Chile. Encuesta de Caracterización Socioeconómica Nacional. Chile; 2000.

F Se agruparon las siguientes respuestas: por intervención quirúrgica, por enfermedad que requirió tratamiento médico, por accidente que requirió intervención quirúrgica y por accidente que requirió sólo tratamiento médico. 
Tabla 5. ¿Qué grado de satisfacción obtavo por la atención recibida durante su última hospitalización, en la infraestructura en general?

\begin{tabular}{|c|c|c|c|c|c|c|c|c|}
\hline & & Trabajac & fo & rmal & & Tra & ajador info & ormal \\
\hline & & oporción & & $\operatorname{rror}^{(1)}$ & Desviación ${ }^{(2)}$ & Proporción & $\operatorname{Error}^{(1)}$ & Desviación ${ }^{(2)}$ \\
\hline 1 Muy bueno & $($ nota 7,0$)$ & $36,9 \%$ & \pm & $2,8 \%$ & $2,5 \%$ & $30,4 \%$ & $\pm 2,5 \%$ & $2,6 \%$ \\
\hline 2 Bueno & (nota 5,5$)$ & $53,7 \%$ & \pm & $2,9 \%$ & $1,9 \%$ & $60,1 \%$ & $\pm \quad 2,7 \%$ & $1,7 \%$ \\
\hline 3 Regular & $($ nota 4,0$)$ & $5,5 \%$ & \pm & $1,3 \%$ & $2,5 \%$ & $6,1 \%$ & $\pm 1,3 \%$ & $2,6 \%$ \\
\hline 4 Malo & $(\operatorname{nota} 2,5)$ & $1,6 \%$ & \pm & $0,7 \%$ & $2,8 \%$ & $1,9 \%$ & $\pm \quad 0,7 \%$ & $2,9 \%$ \\
\hline 5 Muy malo & $($ nota 1,0$)$ & $0,9 \%$ & \pm & $0,6 \%$ & $3,9 \%$ & $0,1 \%$ & $\pm 0,2 \%$ & $3,1 \%$ \\
\hline 6 No sabe & & $1,4 \%$ & \pm & $0,7 \%$ & $3,7 \%$ & $1,5 \%$ & $\pm 0,7 \%$ & $2,4 \%$ \\
\hline Nota promedio & & 5,9 & \pm & 0,059 & 1,029 & 5,8 & $\pm 0,051$ & 0,920 \\
\hline
\end{tabular}

(1) El margen de error se estimó con un nivel de significancia de $95 \%$.

(2) Desviación estándar poblacional.

con nota 5,9 , donde las notas varían entre 1 y 7 .

- En cuanto a la satisfacción con el trato del personal administrativo, un $35,5 \%$ de $\operatorname{los}$ trabajadores formales manifestó que el trato del personal administrativo fue "Muy bueno", en cambio sólo un 31,2\% de los trabajadores informales manifestó que el trato del personal administrativo fue "Muy bueno".

- Un 36,9\% de los trabajadores formales manifestó que la infraestructura es "Muy buena", en cambio sólo un 30,4\% de los trabajadores informales manifestó que la infraestructura es "Muy buena".

\section{CONCLUSIONES}

La desprotección a la que, por definición, están expuestos los trabajadores de la economía informal, afecta su nivel de salud. Es así como, estos trabajadores presentan un menor nivel de salud, menor nivel de acceso a hospitalizaciones e intervenciones quirúrgicas y reciben atenciones de menor calidad, en cuanto a oportunidad, satisfacción con la atención médica, satisfacción con el trato del personal administrativo y satisfacción con la infraestructura.

Un $58,4 \%$ de los trabajadores informales, aproximadamente 1.404 .992 trabajadores, tendría una cobertura inadecuada y parcial de sus necesidades de seguridad social derivadas de accidentes laborales. Lo inadecuado reside en que estos servicios se prestarian en instancias cuyo objetivo es otro, y la parcialidad se debe a la ausencia del subsidio por incapacidad laboral.

Lo anterior, sumado a la mayor exposición que experimentan estos trabajadores a las enfermedades y accidentes del trabajo, determina un espacio, de gran valor para la sociedad, que aún no ha sido satisfecho por la institucionalidad chilena.

Casi un $80 \%$ de los trabajadores informales no tendría cubierta su necesidad de seguridad social para la vejez, invalidez y sobrevivencia.

Por último, un $100 \%$ de los trabajadores informales no tendría cubierta su necesidad de seguridad social, en el caso de desempleo y circunstancias familiares.

\section{REFERENCIAS}

1.- ESPINOZA H. Protección social en salud para trabajadores del sector informal urbano: conceptos, mecanismos y una propuesta. CEDEP. Septiembre, 2004. [Consultado el 23 de Noviembre de 2004]. Disponible en: www.consorcio.org/novedades/ RiosEspinoza.pdf

2.- KANNAN K P. The welfare fund model of social security for informal workers: The Kerala experience. Working Paper 332. Centre for Development Studies. April 2002. [Consultado el 23 de Noviembre de 2004]. Disponible en: http:// unpan l.un.org/intradoc/groups/public/documents/ APCITY/UNPAN010720.pdf

3. LOAYZA $N$ V. The economics of the informal sector, a simple model and some empirical evidence from Latin America. The World Bank [en linea]. Washington D.C. [Consultado el 23 de Noviembre de 2004]. Disponible en: www.worldbank.org/html/ dec/Publications/Workpapers/WPSI700series/ 
wps 1727/wps 1727.pdf

4.- LUND L, SRINIVAS S. Learning from experience: a gendered approach to social protection for workers in the informal economy. STEP and WIEGO. 2002. [Consultado el 23 de Noviembre de 20041. Disponible en: www.ilo.org/public/spanish/ protection/socsec/step/download/24pl.pdf

5.- Oficina de Actividades para los Trabajadores, OIT. Los sindicatos y el sector informal: en pos de una estrategia global. OIT [en línea]. Ginebra, Octubre de 1999. [Consultado el 6 de Octubre de 2004]. Disponible en: www.ilo.org/public/spanish/region/ ampro/cinterfor/temas/worker/doc/otros/iv/i/ index.htm

6.- OIT. Convenio sobre la seguridad social (norma mínima), 1952 (núm. 102). [Consultado el 23 de Noviembre de 2004]. Disponible en: www. stps.gob.mx/01_oficina/03_cgai/convenios/ c102.htm

7.- OIT. El trabajo decente y la economía informal. Informe VI. Conferencia Internacional del Trabajo, $90^{\mathrm{a}}$ reunión. OIT [en línea].Ginebra, 2002. [Consultado el 6 de Octubre de 2004]. Disponible en: www.ilo.org/public/spanish/standards/relm/ilc/ ilc90/pdf/rep-vi.pdf

8.- OIT. Informe sobre el trabajo en el mundo 2000: La seguridad de los ingresos y la protección social en un mundo en plena transformación. 2000. [Consultado el 23 de Noviembre de 2004]. Disponible en: www.ilo.org/public/spanish/bureau/ inf/pkits/

9.- OIT Síntesis de Estudios de caso de Microseguros y otras modalidades de protección social en salud en América Latina y el Caribe. México. 1999. wwwilo-mirror.cornell.edu/public/english/protection/ socsec/step/download/sintesis.doc

10.- ROTH J. Informal Micro-Finance Schemes: the case of funeral insurance in South Africa. Working Paper $N^{\circ} 22$. Social Finance Unit ILO . 2001. [Consultado el 23 de Noviembre de 2004]. Disponible en: www.ilo.org/public/english/employment/finance/ download/wpap22.pdf

11. - SOCIAL ALERT. Economía Informal. Social Alert, serie Estudios sobre Derechos Económicos, Sociales y Culturales len línea]. [Consultado el 23 de Septiembre de 2004]. Disponible en: www. socialalert.org/pdf/2308brochureSpred.pdf

12.- TENDLER J. Small firms. The informal sector and the devil's deal. MIT Cambridge, Massachusetts, IDS bulletin, vol. $33, n^{\circ} 3$, Julio de 2002 . [Consultado el 23 de Noviembre de 2004]. Disponible en: Www.oecd.org/dataoecd/43/41/2489858.pdf

13.- VAN GINNEKEN W. Seguridad social para el sector informal: un nuevo desafío se presenta a los países en desarrollo. Revista Internacional de Seguridad Social [en línea], vol. 52, núm. 1, 1999. [Consultado el 23 de Noviembre de 2004]. Disponible en: www.ilo.org/public/spanish/ standards/relm/gb/docs/gb276/esp-4-1.htm

14.- ESPINOSA J C, HERNÁNDEZ Y W D. Savedoff Productivity and health status in Nicaragua.

15. - MESA-LAGO C. Protection for the informal sector in Latin America and the Caribbean, en V. E. Tokman (director de la publicación) Beyond regulation: The informal economy in Latin America (Boulder, Colorado, Lynne Rienner Publishers, 1992).

16. - Ministerio de Planificación y Cooperación, Gobierno de Chile. Encuesta de Caracterización Socioeconómica Nacional. Chile; 2000.

17.- OIT. Recomendación sobre la seguridad de los medios de vida, 1944 (núm. 67).

18.- OIT. Recomendación sobre la asistencia médica, 1944 (núm. 69).

19 SÁNCHEZ M, LABBÉ J. El sector informal en Chile: una visión estadística. Santiago de Chile. 2004.

20.- Superintendencia de Isapres. Series estadísticas sistema isapre 1981-2001. Santiago, Superintendencia de Isapres: 2002.

21.- VAN GINNEKEN W. Social protection for workers in the informal economy: New challenges for Asia and the Pacific. Twelfth Regional Conference for Asia and the Pacific, Towards more effective social security in Asia and the Pacific, Bangkok, 20-23 November 2000. Geneva, ISSA. 2000.20 p.

Usted puede comentar éste y otros artículos publicados en la Revista Chilena de Salud Pública, enviando un correo electrónico a revistasp@ med.uchile.cl 